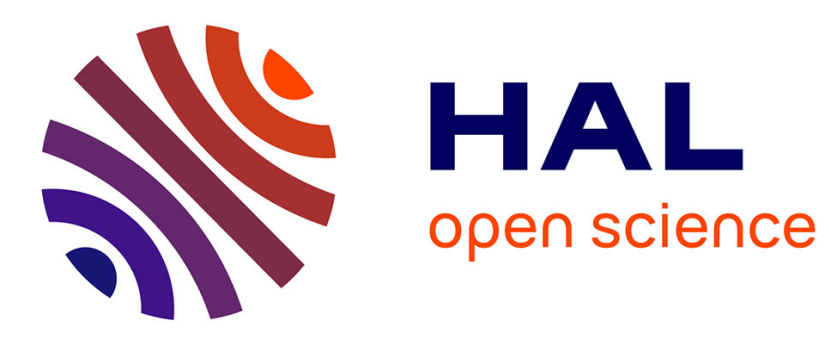

\title{
Le tourisme comme expérience sensible
}

Bule Liu, Luc Gwiazdzinski, Wenbo Hu

\section{To cite this version:}

Bule Liu, Luc Gwiazdzinski, Wenbo Hu. Le tourisme comme expérience sensible. Teoros. Revue de recherche en tourisme, 2019. hal-02128500

\section{HAL Id: hal-02128500 \\ https://hal.science/hal-02128500}

Submitted on 14 May 2019

HAL is a multi-disciplinary open access archive for the deposit and dissemination of scientific research documents, whether they are published or not. The documents may come from teaching and research institutions in France or abroad, or from public or private research centers.
L'archive ouverte pluridisciplinaire HAL, est destinée au dépôt et à la diffusion de documents scientifiques de niveau recherche, publiés ou non, émanant des établissements d'enseignement et de recherche français ou étrangers, des laboratoires publics ou privés. 


\title{
Téoros
}

Revue de recherche en tourisme

38, 1 | 2019:

Tourisme urbain

Hors thèmes

\section{Le tourisme comme expérience sensible}

\section{Première approche des ressentis des touristes chinois en France à partir des données issues des réseaux sociaux numériques}

\author{
Bule Liu, Luc Gwiazdzinski et Wenbo Hu
}

\section{Résumé}

Le développement du tourisme oblige les acteurs et les territoires à s'adapter à la demande et aux besoins des visiteurs originaires de différents pays et cultures. Cette exigence interculturelle passe notamment par une connaissance plus fine des pratiques, des perceptions et des ressentis des touristes étrangers et notamment des touristes chinois, dont le nombre est en forte augmentation en France, en Europe et dans le monde. L'article propose une approche exploratoire des représentations associées à ces pratiques en France par l'exploitation des commentaires et des images postés sur le réseau social Sina Microblog (Weibo) par des touristes chinois en visite dans la ville d'Avignon à l'été 2016 et aboutit notamment à une première typologie des ressentis. Le protocole d'analyse des pratiques touristiques développé pour cet exemple permet d'appréhender le niveau de satisfaction des touristes, d'analyser ce qui forge leur comportement complexe à partir de leur ressenti sur place, de mieux caractériser l'expérience touristique au sens large et d'imaginer une modélisation. D'un point de vue plus opérationnel, la démarche permet d'envisager une amélioration du dialogue entre les opérateurs touristiques et les usagers finaux, une adaptation plus fine de l'offre et de la demande, une amélioration du bien-être des touristes et un renforcement de l'attractivité des territoires.

\section{Texte intégral}

De 2006 à 2016, le nombre de touristes interculturels dans le monde est passé de o,85 milliard à 1,2 milliard (Banque mondiale, n.d.). Cet essor nécessite une adaptation accélérée et fine de l'offre touristique à une demande complexe qui dépasse les approches traditionnelles en cherchant à comprendre ce qui forge le comportement complexe des touristes à partir de leur ressenti sur place. 


\section{Forte augmentation du tourisme chinois}

2 Cette préoccupation concerne notamment les touristes chinois dont le nombre est en forte augmentation avec d'importants enjeux en matière de développement économique. Selon l'agence Atout France (2016), on a dénombré 1,66 million de touristes chinois en France en 2014 pour un nombre moyen de 6,6 nuitées. Outre Paris, ses monuments et ses boutiques de luxe, le Sud de la France est attractif, en partie grâce aux traductions du livre de Peter Mayle Une année en Provence (1994) et d'une minisérie télévisée chinoise Dreams Link1, filmée au milieu des champs de lavande du midi sur fond de citadelles fortifiées. Ces touristes chinois dépenseraient en moyenne $3400 €$ par personne pour leur séjour, dont $30 \%$ (1000 €) pour la consommation d'articles de luxe, $15 \%$ (500 €) pour l'hébergement et $3 \%$ (102 €) seulement pour les activités de loisirs (visites guidées, sports...).

\section{Décalage entre l'offre et l'attente}

Les témoignages d'expériences sur les réseaux sociaux et dans les médias² mettent régulièrement en exergue des décalages importants entre l'offre touristique actuelle (restauration, hôtellerie...) et les besoins des touristes chinois qui ne sont pas toujours satisfaits. Une enquête 3 auprès des voyageurs chinois a révélé que pendant leurs vacances sur le continent, $46 \%$ d'entre eux n'avaient mangé qu'une seule fois des aliments « européens » et 10 \% pas du tout. Il paraît nécessaire de dépasser ce constat pour chercher à comprendre les éléments qui influencent ce ressenti et les comportements associés.

\section{Décalage entre pratiques et besoins}

Les touristes chinois seraient attirés dans un premier temps par les hauts lieux touristiques où ils aiment se photographier (Atout France, 2016). Parmi leurs activités, le shopping occupe la première place avec un budget plus important que pour l'hébergement ou la restauration. Ils y consacrent au moins une demi-journée pour les achats et une soirée pour un spectacle de type cabaret. Dans les pré-enquêtes menées en 2016 auprès de ces touristes, nous avons pu constater que ces informations correspondaient plus aux activités effectives des touristes en France qu'à des besoins et des attentes réels en décalage avec l'offre des acteurs et des territoires français. Contrairement aux idées reçues, le passage par le shopping de luxe proposé par la plupart des agences n'est pas indispensable. Autre élément à prendre en compte, le comportement touristique évolue vers davantage d'individualisme, surtout parmi les jeunes (18 - 35 ans), qui représentent 60 \% des Chinois qui voyagent dans le monde.

\section{Nécessaire connaissance des besoins}

$\mathrm{Au}$-delà des enquêtes classiques de fréquentation et de satisfaction, on peut constater une relative méconnaissance des envies, des besoins et des attentes des touristes. Dans un contexte de développement rapide de cette activité, une approche interculturelle et sensible est nécessaire afin d'adapter l'offre des pays visités à une demande venue d'autres continents et cultures. Pour les acteurs du secteur comme pour les chercheurs, il est temps de poser un autre regard sur les comportements touristiques en étudiant notamment les sentiments et les ressentis des «populations présentes » (Gwiazdzinski et Klein, 2014 ; Hu et al., 2016).

\section{Importance de la notion d'expérience touristique}


6 Les lacunes observées obligent également à aborder le tourisme comme une « expérience sensible » (Agapito et al., 2013), un processus complexe d'interactions dans lequel les dimensions sentimentale et émotionnelle (Bonnin, 2002 ; Ladwein, 2002 ; 2003) doivent être prises en compte. Dans ce cadre, les notions « d'expérience » (Batat et Frochot, 2013), de " perception » (de Souza Bispo, 2016), voire " d'immersions dans des expériences variées » (Firat, 2001) et "incorporées » (Thompson, 1998), développées dans les domaines du marketing et de la géographie, sont centrales pour comprendre les motivations profondes des comportements touristiques.

\section{Nouvelle piste d'exploration à partir des réseaux sociaux}

Nous proposons d'explorer une nouvelle voie par l'exploitation de données issues des réseaux sociaux et qui permettent la prise en compte des émotions (" réactions affectives transitoires habituellement provoquées par une simulation venue de l'environnement » [Larousse]) des visiteurs in vivo et une meilleure compréhension de l'expérience touristique sensible à partir du recueil et de l'exploitation de leurs réactions et publications directes en ligne. Au-delà, cette première approche questionne les formes « d'adaptation » du tourisme au sens général de " processus par lequel un être ou un organe s'adapte naturellement à de nouvelles conditions d'existence », mais aussi l'adaptation du touriste au sens de la « modification des fonctions psychiques de l'individu qui, sans altérer sa nature, le rendent apte à vivre en harmonie avec les nouvelles données de son milieu ou un milieu ouvert »4.

La recherche exploratoire engagée - s'appuyant sur des concepts empruntant à différentes disciplines - propose d'étudier les comportements d'un échantillon de 413 touristes chinois ayant séjourné pendant l'été 2016 dans la ville touristique d'Avignon dans le Sud de la France. Elle est construite sur l'observation et le traitement des commentaires et des images postées sur le réseau social numérique chinois le plus populaire : Sina Microblog (Weibo).

9 Sur la base d'une première typologie comportementale des touristes (Carù et Cova, 2003), cet article propose un cadre d'observation et d'interprétation du processus d'interaction entre les touristes et la destination touristique selon leurs émotions, leurs réactions et leurs comportements.

\section{Un contexte et des pratiques en mutation}

Les recherches engagées s'inscrivent dans un contexte de mutation des comportements - comme "manière d'être d'agir ou de réagir des êtres humains, d'un groupe » (Larousse) - des touristes et de l'offre touristique que l'on peut définir comme l'ensemble des services et des biens finaux proposés au consommateur par le secteur touristique.

\section{Interaction participative : vers un tourisme « pratique »}

11 Les comportements touristiques ont changé même si des pratiques de masse de ce que l'on pourrait qualifier de " crypto-tourisme » persistent en parallèle (GravariBarbas et Delaplace, 2015). 


\section{Contexte d'hybridation de l'offre et des comportements touristiques}

12 L'ancien rapport au tourisme, dit " rapport d'ici-ailleurs » (Bourdeau et Bensahel, 2013) où les touristes voyagent (" ailleurs ») en cherchant l'exotisme et la différence avec leur vie quotidienne et leur environnement habituel («ici ») (de Souza Bispo, 2016), a changé. Ce rapport « classique », en lien avec la notion de tourist gaze (Urry, 1992) marquée par la constitution du tourisme en termes "d'extraordinaire » de la destination par rapport à l'ordinaire de la « vie quotidienne » (De Certeau, 1980), n'est plus le même.

On assiste à une hybridation croisée. D’un côté, on voit émerger un " exotisme de proximité » avec le développement d'offres de découverte de lieux inconnus et insolites5, de parcours thématiques qui proposent de poser « un autre regard » sur sa propre ville6, la banalisation des pratiques d'exploration urbaine (urbex) et des activités touristiques qui se localisent près de chez soi (Bourdeau et Bensahel, 2013). D'un autre côté, les activités et les éléments de la vie quotidienne (repas traditionnels, habitudes...) sont pratiqués dans la destination touristique, consciemment ou non (Edensor, 2007). Les touristes maintiennent des sociabilités habituelles (échanges avec amis...) en voyage (Larsen, 2008). Ils choisissent souvent la plateforme de location AirBnB, pour pouvoir continuer à cuisiner leur nourriture habituelle. Lors de nos déplacements, nous avons souvent croisé des touristes chinois pratiquant leurs jeux habituels (comme le mahjong) et même un groupe voyageant avec des valises entières de viande cuisinée selon ses habitudes.

14 Au-delà de ces premières observations ponctuelles, ces questions encore peu explorées nécessitent de nouvelles investigations intéressantes par les acteurs du secteur touristique confrontés à ces décalages, les pouvoirs publics qui gèrent les villes et les territoires.

\section{Une approche du tourisme axée sur l'expérience et la pratique}

Avec l'évolution du rapport ici-ailleurs (Bourdeau et Bensahel, 2013), les binômes « ici-ailleurs », " visiter-habiter », « travail-vacances »... ont tendance à se confondre et les statuts à s'hybrider (Gwiazdzinski, 2016). Il est de plus en plus difficile de définir l'espace (en tant que destination) et le temps (en tant que temps de loisir) du tourisme. Il est donc difficile de qualifier les activités touristiques par une simple mesure de l'espace (distance) et du temps (durée) des touristes et il faut s'appuyer sur une autre approche des comportements et des motivations en immersion.

Il est important de prendre en compte les « pratiques touristiques » comme une série d'activités, d'échanges et d'interactions (Gherardi, 2009), ou encore d'« ambiances » (Augoyard, 2010) au lieu de destination. Le comportement est lié à un ensemble de stimulants externes et d'irritabilités internes, d'interactions continuelles avec les objets, qui fournissent une séquence des réactions ou de manifestations comportementales ou/et mentales motivant le besoin en affectant les pensées et les comportements à travers des changements somatiques et psychologiques suscités par les interactions continuelles avec les objets (de consommation) : colère, crainte, mépris, dégoût, embarras, plaisir, excitation, peur, gratitude, culpabilité, intérêt, ressentiment, tristesse, honte, surprise et vertu (Gherardi, 2009). Les pratiques sont des coproductions des stimulants externes (caractère spécifique des objets, environnement, comportement, dialogue, couleur, etc.) et des irritabilités internes (réactions mentales et comportementales).

" L’immersion expérientielle » est un état d'activité intense dans lequel le consommateur se trouve quand il accède pleinement à l'expérience. L'activité comporte un ou plusieurs types de manifestations : cognitive, émotionnelle (affective), physique, perceptuelle (sensorielle) et sociale (Fornerino et Helme-Guizon, 2006). Intégrant la notion d'« expérience » dans le champ du tourisme pour l'étude d'une station de ski, 
Isabelle Frochot et Dominique Kreziak (2009 : 10) ont montré l'importance de cette coproduction et de ces interactions continues et cumulatives entre les lieux touristiques et les touristes : « Le rôle de la station est d'assurer la fluidité du service, en apportant un éventail d'activités sur la base desquelles les clients vont construire leur propre expérience et leur propre vécu de consommation ». Ils ont insisté sur la « coconstruction de l'expérience de consommation touristique, où prestataires de service et touristes collaborent pour créer le maximum de valeur dans le séjour ».

Dans notre étude, le tourisme est considéré comme un processus sensible, une séquence de comportements et de pratiques avec des affections, qui jouent un rôle essentiel dans les caractéristiques subjectives et symboliques attribuées à l'offre touristique.

La problématique de la production du tourisme pose également la question inverse, c'est-à-dire comment le comportement touristique est-il influencé par la structure sensible? Plusieurs études sur le tourisme sportif ou d'aventure (entre autres Arnoul et Price, 1993 ; Mackenzie et Kerr, 2013) font ressortir que " les résultats émotionnels associés à une expérience extraordinaire sont intégrés dans les relations entre le client et le fournisseur de services7 ». Dora Agapito, Júlio Mendes et Patrícia Valle (2013) ont pour leur part montré que l'information multisensorielle concernant les expériences touristiques était importante dans le marketing et la gestion des destinations et que l'analyse de la dimension sensorielle des expériences touristiques était pertinente : les sens humains sont cruciaux pour la perception du monde par l'individu ; les stimuli sensoriels influencent le comportement du consommateur et les lieux et les environnements (destinations) offrent des rencontres multi-sensorielles. Pour Jürgen Gnoth et Xavier Matteucci (2014 : 5), l'expérience du touriste (TEM Tourism Experience Model) serait « le mélange de ce qui perçu par les sens, son traitement et la manière dont il est retenu dans l'expérience qui en résulte ». En décrivant les processus interreliés par lesquels les êtres humains acquièrent, modifient et adaptent les connaissances et les compétences en fonction de leur being-in-the-World (être au Monde) émotionnel, on peut réussir à comprendre comment les touristes structurent et perçoivent leur destination. Pour Gnoth et Matteucci, (2014: 6), le TEM pourrait être décomposé en une typologie d'expériences sensibles en quatre modes : «l'expérience comme pur plaisir, comme re-découverte, comme exploration existentiellement authentique et comme recherche de connaissance ».

Silvia Gherardi (2009), quant à elle, avance que le concept de " pratique » contribue à interpréter la manière dont les gens atteignent le being-in-the-world 8 . Ces «pratiques » ne sont pas seulement des « patterns » (ou schémas) récurrents d'actions productives, mais également des actions socialement durables qui reproduisent la société. En suivant ce concept, les pratiques du tourisme - qui rejoignent l'étude « Tourism as Practice » de Marcelo de Souza Bispo (2016) - peuvent être identifiées et segmentées par leur liaison aux quotidiens, autant que par leur fonctionnement dans les contextes physiques et par les contacts sociaux et humains.

Sur la base de ces différents travaux, nous proposons d'organiser la réflexion autour de quatre types principaux de pratiques du tourisme : pratiques de vie, pratiques d'exploration et d'apprentissage, pratiques de sociabilité, pratiques d'interactions (voir tableau 1). En ce sens, le tourisme n'apparaît plus comme une activité « dominée » par l'offre, mais plutôt une « coproduction » entre touristes et milieux locaux. « Ce que nous sentons, ce que nous souhaitons et ce que nous pensons sont en totale harmonie. » (Frochot et Kreziak , 2009)

Tableau 1 : Quatre types de pratiques du tourisme

\begin{tabular}{|l|l|l|}
\hline $\begin{array}{l}\text { Les pratiques comme une } \\
\text { série d'activités qui.... }\end{array}$ & Lien au quotidien permanent & $\begin{array}{l}\text { Pratiques purement exotiques et } \\
\text { touristiques }\end{array}$ \\
\hline $\begin{array}{l}\text { Fonctionnent par rapport } \\
\text { au contexte physique }\end{array}$ & $\begin{array}{l}\text { Pratiques de vie (se déplacer, dormir, } \\
\text { suivre des ateliers...) }\end{array}$ & $\begin{array}{l}\text { Pratiques d'exploration et } \\
\text { d'apprentissage (visiter, faire } \\
\text { l'expérience des attractivités, } \\
\text { connaître...) }\end{array}$ \\
\hline $\begin{array}{l}\text { Fonctionnent par rapport } \\
\text { aux contacts sociaux }\end{array}$ & $\begin{array}{l}\text { Pratiques de sociabilité (partager } \\
\text { entre amis, s'occuper de la famille, } \\
\text { contacter...) }\end{array}$ & $\begin{array}{l}\text { Pratiques d'interactions (parler avec les } \\
\text { locaux, suivre le guide, apprendre...) }\end{array}$ \\
\hline
\end{tabular}


Notre recherche sur les touristes chinois s'appuie donc sur des indicateurs d'intégration (facteurs procéduraux, facteurs expérientiels) entre touristes et destinations (lieux, personnes, ambiances). Dans un premier temps, nous appuyant sur des observations préalables sur le terrain et sur le réseau social Sina Microblog (Weibo), nous avons identifié des éléments spécifiques influençant l'expérience des parcours touristiques individuels. Dans un deuxième temps et sur la base de ces indicateurs, nous avons classé manuellement les commentaires et les photos que les touristes partagent sur le réseau social afin de comprendre l'expérience sensible de chaque touriste, comment ils pratiquent les espaces et les temps d'Avignon, ainsi que leurs ressentis.

\section{Une approche méthodologique}

Notre étude exploratoire permet de mesurer un certain degré d’intégration entre les touristes et les destinations au sens du lieu, des personnes et des ambiances. Dans un premier temps, nous cherchons à identifier des éléments spécifiques dans l'expérience des parcours individuels des touristes. Dans un deuxième temps, nous les classons en fonction des différents moyens qu'ils mettent à contribution pour s'immerger et s'approprier les espaces et les temps.

\section{Le choix d'une population, d'un réseau social et d'un territoire}

24 Notre recherche porte sur 413 échantillons de commentaires provenant de touristes chinois en voyage dans la ville d'Avignon que nous avons pu collecter sur Sina Microblog (Weibo) pendant l'été 2016.

\section{Un réseau populaire et accessible pour des touristes chinois en augmentation}

Notre choix d'étudier des touristes chinois s'appuie notamment sur l'importance et l'accroissement rapide des flux de touristes en provenance de ce pays - notamment vers la France qui est leur premier pays d'accueil -, sur une certaine méconnaissance que nous avons relevée à propos des comportements de ces populations et sur notre compréhension culturelle et linguistique qui facilite la collecte et l'exploitation des données.

Nous avons retenu le réseau Sina Microblog (Weibo) car c'est actuellement le plus populaire en Chine. Les informations disponibles y sont également beaucoup plus riches que la seule trace individuelle d'un téléphone portable utilisée dans d'autres recherches (Lucchini et al., 2013 ; Gwiazdzinski et Drevon, 2014) : informations personnelles sur les usagers, localisation dans l'espace et dans le temps, ressentis, sentiments, réactions, perceptions, échanges et interactions. Cette diversité répond bien aux besoins de notre étude sur l'expérience touristique. En outre, par rapport à d'autres réseaux sociaux tels que Wechat, les données sur Sina Microblog (Weibo) sont accessibles et ouvertes au public : nul besoin de se connecter pour suivre les commentaires.

Par ailleurs, ce sont les utilisateurs qui alimentent volontairement ce réseau en informations. Concrètement, les personnes en situation y publient des informations à partir de leur smartphone (image, texte...). Leurs expériences touristiques localisées dans l'espace et dans le temps (minute, jour...), sous la forme de photos et de commentaires - y compris des échanges d'informations pratiques ou/et des ressentis concernant les lieux visités -, sont automatiquement géo-référencées et visibles par tous sans obligation de devenir membre. 


\section{Une ville touristique attractive}

Le choix d'Avignon est lié à l'attractivité et à la réputation au-delà des frontières du site touristique et de son festival - complet du 6 au 24 juillet 2016 (AFP, 2016) - et au grand nombre de touristes chinois détectés sur place à travers ce réseau social en juillet 2016, période de vacances pour les jeunes chinois. D'après les acteurs touristiques interrogés sur place, l'attractivité de la région d'Avignon auprès des touristes chinois serait attribuable aux champs de lavande, aux événements culturels internationaux (festival de théâtre...) et à la proximité de grands sites naturels comme le Verdon.

\section{La recherche d'indicateurs comportementaux}

La littérature fait abondamment état d'éléments factuels sur le comportement touristique (consommation, destination, durée, dépenses...), mais elle est moins prolixe sur les sentiments et les ressentis in vivo des individus immergés. Cette ignorance en partie liée aux difficultés (méthodologiques et techniques) d'acquisition d'indicateurs comportementaux peut laisser s'installer une discordance entre l'offre et le besoin touristique dommageable pour le secteur touristique et pour les échanges interculturels. À partir d'une approche "sensible » (Sansot, 1985), nous proposons de mettre en évidence les modes d'interaction et de dialogue entre touristes immergés et milieu.

\section{Première typologie de l'interaction touriste-destination}

D'abord, nous ne voyons plus le tourisme comme une dominance de l'offre mais comme une coproduction entre touristes et milieux locaux. C'est ce que nous définissons comme les " pratiques » et les interactions entre touristes et destinations. L'expérience, cette « connaissance ou ce savoir-faire acquis par la pratique, hors d'un enseignement théorique ", est la part la plus importante produite par un comportement touristique (Revue Espace, 2014 : 2). C'est à la fois la plus mémorable et la plus mémorisée par les différents acteurs.

Sur ces bases, nous proposons une première typologie permettant d'identifier l'expérience touristique à travers des croisements entre les « facteurs procéduraux » et les « facteurs expérientiels » des touristes (voir tableau 2). Ce cadre contient différents critères expérientiels : 1) des facteurs procéduraux au cours des comportements touristiques (nidification, exploration et marquage), et 2) des facteurs expérientiels (physique, effectif, cognitif, sensoriel, social, référentiel et réflexe).

Tableau 2 : Typologie expérientielle de pratiques touristiques

\begin{tabular}{|l|l|l|l|}
\hline & \multicolumn{1}{|c|}{ Nidification } & Exploration & Marquage \\
\hline Physique & & & \\
\hline Affective & & & \\
\hline Cognitive & & & \\
\hline Sensorielle & & & \\
\hline Sociale & & & \\
\hline Référentielle & & & \\
\hline Réflexive & & & \\
\hline
\end{tabular}

Réalisation : les auteurs

\section{Facteurs procéduraux}

Nous appuyant sur la théorie de l'appropriation d'espace, nous nous interrogeons, d'une part, sur « l'exercice d'une autorité, d'un contrôle et d'un pouvoir (physique ou/et psychologique) sur un lieu » (Prohansky et al. 1970) et, d'autre part, sur « l'intervention sur un espace pour le transformer et le personnaliser » (Fischer, 1992) en « un chezsoi » (Aubert-Gamet, 1996). 
33 Antonella Carù et Bernard Cova (2006) ont développé une analyse de l'expérience touristique - dans des contextes différents de magasins, d'usines, de fêtes, de sites Internet, de récits - et mis en évidence trois types de comportements entre les lieux et des touristes qui constituent un modèle d'appropriation dans l'expérience que nous proposons de mobiliser pour l'étude de l'expérience touristique des visiteurs chinois à Avignon.

34 Les facteurs procéduraux concernent les manières dont les touristes interagissent avec les objets touristiques selon trois modes d'appropriation : la nidification, l'exploration et le marquage. Ces phases sont repérées à partir des indications exprimées par les touristes dans leurs messages sur les réseaux numériques sociaux.

35 La «nidification » correspond à une première phase de visite. Les touristes arrivent à destination ; ils construisent une enclave familiale adaptée à leurs habitudes quotidiennes, comme chez eux. Cette « enclave » correspond au logement où ils déposent leurs valises et où ils passeront leur séjour : appartement, hôtel. C'est la création d'un " chez-soi » (Fischer, 1983). Ils « font leur nid », comme on peut le constater dans les extraits de commentaires du groupe de touristes en visite à Avignon :

[N]ous sommes finalement arrivés à la maison [hôtel], j'ai du temps pour ranger mes valises et regarder les réseaux sociaux.

$[\mathrm{N}]$ ous prenons un petit déjeuner chinois comme d'habitude...

38 L' ' exploration » constitue la deuxième phase de la visite. Les touristes sortent de leur enclave nidifiée et amorcent leur exploration. Ils découvrent les attractions touristiques et commencent à les interpréter en fonction de leur culture et à les qualifier. Cette phase « permet de recevoir les informations et ainsi d'amorcer l'interprétation de lieu » (Fischer, 1981) :

39 On a la chance de découvrir les spectacles du festival d'Avignon. C'est magnifique. J'aimerais vivre dans cette ambiance.

40 Les acteurs sont dans les rues d'Avignon... On danse, on rit...

41 Le « marquage » correspond à la troisième et dernière phase de la visite. Les touristes complètent leur exploration et accumulent assez d'informations sur les attractions. Ils essayent d'interpréter ce qu'ils vivent et de tirer des premières conclusions sur la destination et sur le voyage. Cette conclusion est « marquée » et présentée de manière subjective. Le touriste « intègre son inscription personnelle dans cet espace » (Fischer, 1983) et son impression avec un sens particulier :

$[\mathrm{C}]$ haque personne possède sa propre image de la lavande dans le cerveau mais tout le monde a une sensation de romantisme et d'amour.

[J]e suis dans le rêve de... [avec des photos « photoshoppées »].

\section{Facteurs expérientiels}

$44 \quad$ Notre analyse du degré d'immersion et d'intégration entre touriste et destination est réalisée à l'aide d'une grille de facteurs expérientiels intégrant les cinq facteurs classiques mis en avant par différents auteurs (Carù et Cova, 2006) et deux facteurs supplémentaires basés sur nos observations touristiques en amont de la recherche, soit, au final, les facteurs physique, affectif, cognitif, sensoriel, social, référentiel et réflexif. Nous cherchons à les repérer à partir des interprétations et des expressions personnelles des comportements issus des témoignages sur le réseau social numérique.

L'aspect « physique » inclut toutes les activités physiques que les touristes effectuent directement sur place dans le territoire et leurs contacts avec la société locale, ou indirectement par le biais de leur clavier :

47 [F]inalement, j'ai fini à PS [photoshopper] ces photos d'Avignon avant d'arriver à Nice.

L'aspect « affectif » concerne les émotions des touristes. Ces émotions sont fortement liées à leurs expériences personnelles avant le séjour, à leurs rencontres pendant le séjour et à leur degré de satisfaction :

$[J]$ 'en ai marre qu'il n'y ait pas de choses à faire le soir... 
$50 \quad[$ C]ette expérience est super ! Je voudrais retourner en Provence au cours des prochaines années...

51 L'aspect « cognitif » concerne les connaissances ou les informations que les touristes mobilisent afin de confirmer la compréhension du contexte tangible et intangible de la destination. Ces connaissances sont généralement obtenues par l'accumulation des savoirs avant le séjour ou par l’interprétation touristique pendant le séjour :

[E]st très ancien... [quelqu'un a] construit cette ville à... Autour de... [l'attraction], il y a...

C'est la ville natale de la lavande.

54 L'aspect « sensoriel » concerne les sentiments et les sens que les touristes mobilisent à destination. Ces sentiments peuvent être non seulement extérieurs au sens où ils concernent le contexte de la destination, mais aussi intérieurs, quand il s'agit des sensations des touristes eux-mêmes (exemple : « il a froid quand il est malade ») :

Il pleut tous les jours et comme en plus il fait froid, nous restons à la maison.

[La/l'/le] couleur/odeur/parfum/son/goût de lavande est super, surtout.

L'aspect « social » concerne les liens humains et les relations que les touristes développent avec la société locale et les autres visiteurs. Ils sont à relier à d'autres types d'expériences, en particulier les aspects physique et affectif, au contact avec les habitants et les autres visiteurs :

La petite fille m'a offert un sourire doux.

Le personnel nous a accueillis.

L'aspect « référentiel » concerne les « citations » (histoire, expérience personnelle, littérature, etc.) et les comparaisons que les touristes invoquent pour interpréter et juger l'offre touristique. Ces éléments sont également mobilisés pour améliorer l'expérience touristique de manière littéraire et artistique en s'appuyant sur des représentations préalables :

[C]ompter mon expérience touristique avant, c'est la quatrième fois que je choisis une chambre d'hôte...

62 [Ç]a me rappelle l'expérience de jardinage quand j'étais petit...

63 L'aspect " réflexion » se rapporte aux pensées et aux réflexions philosophiques provoquées par l'expérience touristique de la destination pendant le séjour, mais destinées à la vie personnelle hors de la destination et du séjour. Ces pensées et ces réflexions apportent généralement de nouvelles valeurs en termes de transformation et de développement personnel pour le touriste après son séjour sur place :

[Après avoir vu un spectacle de théâtre] concernant l'art, la plus importante chose n'est pas de finir un ouvrage, mais de présenter sa propre émotion, puis de la partager... c'est également la véritable signification de la vie...

[Cette visite] me fait songer à ma décision sur...

\section{Collecte de données sur réseau social numérique et exploitation des données}

66 Le traitement des données a été effectué suivant deux étapes principales : la collecte par le biais de l'analyse du réseau social et la classification selon le protocole de sensibilité consommative que nous avons développé.

67 Cette étude exigeait une diversité d’informations individuelles liées au voyage. Nous référant à l'approche utilisant les informations issues du réseau social (Hu et al., 2016), nous avons collecté les données de Sina Microblog (Weibo) qui comprennent des éléments de localisation dans l'espace et dans le temps, des commentaires descriptifs sur la destination et des réflexions personnelles associées notamment à des facteurs procéduraux et expérientiels.

Au cours de l'été 2016, 1376 commentaires et photos provenant de 413 touristes chinois en voyage dans la ville d'Avignon et faisant état de leur expérience ont été collectés manuellement. Ces commentaires écrits ont été distribués selon des indicateurs préalablement choisis (voir tableau 2). 


\section{Le tourisme comme interaction sensible}

L'exploitation des messages des touristes a permis de mettre en évidence la manière dont les touristes interagissaient avec les objets touristiques dans les trois phases de « nidification », d' « exploration » et de « marquage » (facteurs procéduraux) selon les sept degrés d'intégration (facteurs expérientiels) : physique, affectif, cognitif, sensoriel, social, référentiel et réflexif. Ce traitement des données nous a aidés à dresser un premier tableau des " pratiques touristiques des touristes chinois à Avignon » (voir illustration 1).

\section{Illustration 1 : Interaction des touristes et des objets touristiques}

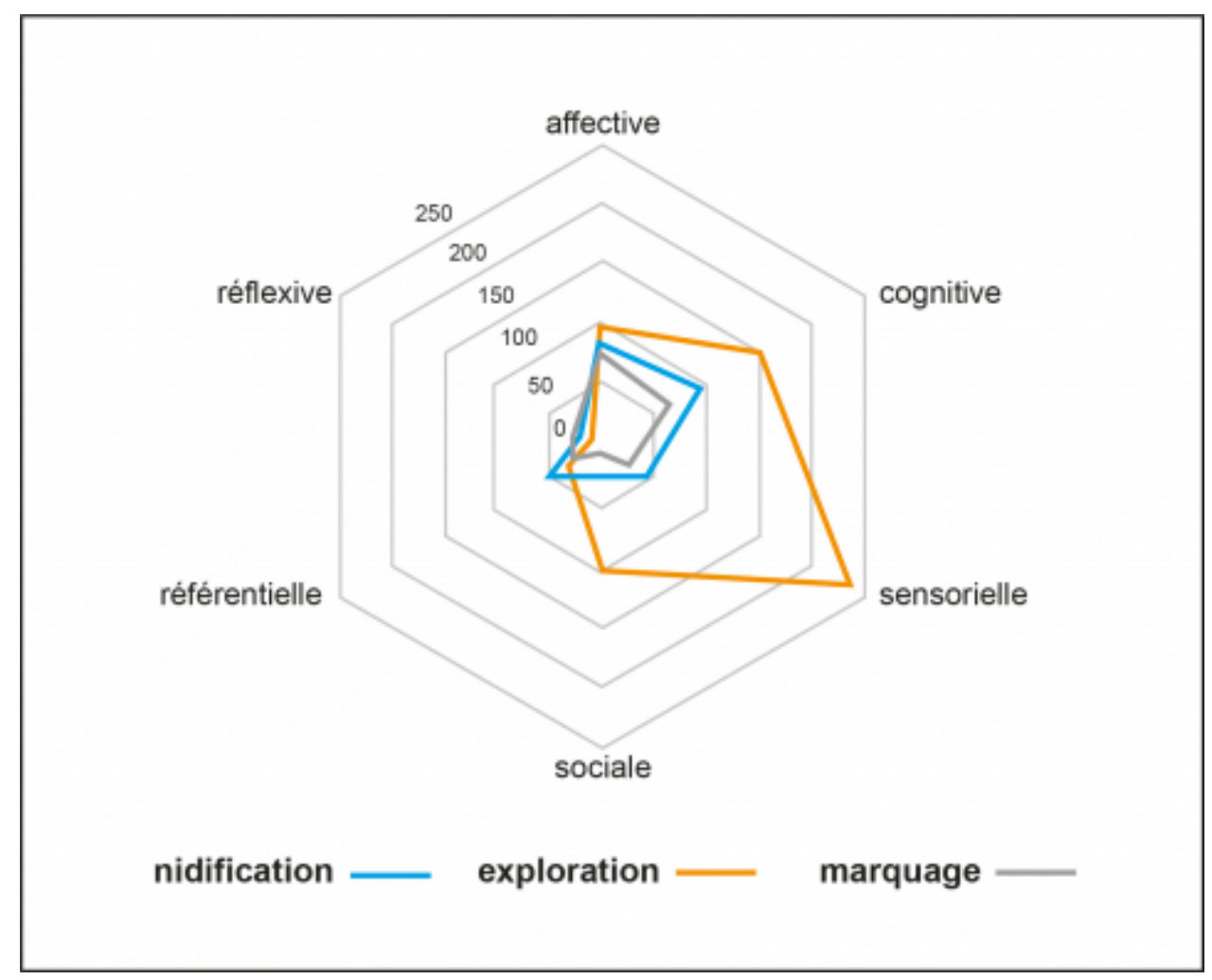

Réalisation : les auteurs

\section{L'expérience touristique en phase de « nidification »}

Dans la phase de « nidification », les touristes se retrouvent dans un nouveau " chezsoi » en construction. Ils insistent fortement sur leurs expériences physiques (25\%), cognitives (23\%) et affectives (21\%). Leurs expériences sensorielles (11\%) et référentielles (11 \%) sont également marquantes. Dans les " récits » touristiques construits sur la toile, les éléments des pratiques touristiques présents se concentrent sur les activités habituelles et quotidiennes dans ce « chez-soi » construit sur place, à destination. Les touristes ont une forte conscience de la "maison », en termes de logement, et de leurs activités dans ce lieu. Sur le réseau, ils décrivent fréquemment le déplacement aller-retour de ce logement vers l'extérieur et la vie quotidienne dans ce « nid » et à proximité.

71 Dans leurs activités quotidiennes, leurs réflexions portent sur les aspects affectifs et cognitifs. En matière d'émotions et de sensations, ils portent attention à eux-mêmes puis au contexte qu'ils construisent. Leurs pratiques et leurs ressentis - repérables dans les commentaires - se situent dans un registre " référentiel ». Ils cherchent en permanence à faire des liens et des comparaisons entre leur situation dans le " chezsoi » construit à destination, leur vie quotidienne et leur expérience passée. Ce phénomène est aussi présent dans leur réflexion et leur sociabilité. En bref, dans cette 
phase de nidification, les touristes chinois s'appuient sur leur expérience pour transformer la destination d'accueil en un « chez-soi ».

\section{L'expérience touristique en phase « d'exploration »}

L'exploration regroupe les éléments que les touristes chinois cherchent à découvrir à destination quand ils sortent de leur « nid ». Dans cette phase d'appropriation touristique, ils ont des expériences sensorielles (32 \%) et cognitives (21\%). Dans leurs commentaires, les touristes se concentrent sur les activités « primaires » du tourisme : visiter et rencontrer. Au cours de l'exploration, ils mobilisent les cinq sens pour qualifier le monde extérieur. Ils cherchent à bien connaître la destination - par rapport aux connaissances imaginées et acquises en Chine -, les histoires et les contextes temporels et spatiaux.

Les éléments sociaux et affectifs sont également remarquables dans leurs récits. Du point de vue social, ils soulignent leurs contacts avec les habitants et les acteurs de la destination. Du point de vue affectif, ils font part d'émotions positives pendant leurs visites. Les éléments de référence et de réflexion sont beaucoup moins fréquents dans cette phase d'appropriation.

\section{L'expérience touristique en phase de « marquage »}

Sur le plan du marquage, les éléments sont présentés comme les ressentis acquis et personnalisés, transformés et appropriés, « à partir de leurs références, de leur histoire personnelle... », afin de construire leur «propre image de la destination ». Dans les commentaires des touristes chinois, ces éléments se lient principalement à leurs expériences affectives (27\%), cognitives (24\%), et physiques (20\%).

Quand nous analysons précisément ces comportements touristiques, nous remarquons que les pratiques touristiques sont largement liées à ce qui est appelé « $P$ selfie » (égo-portrait auto-traité par des applications spécifiques) (voir illustration 2). Tout d'abord, ils prennent des photos comme les autres touristes. Ensuite, avec les applis de leur téléphone intelligent, ils font immédiatement le montage de ces photos en modifiant les couleurs, les structures, même les contenus (par exemple ajouter des éléments d'un dessin animé qu'ils aiment) et marquent leur signature personnalisée sur ces photos, afin d'obtenir un album photographique de cette visite. Enfin, ils publient ces photos modifiées sur les réseaux sociaux pour les partager et échanger.

Illustration 2 : Exemple de $P$-selfie d'un blogue personnel
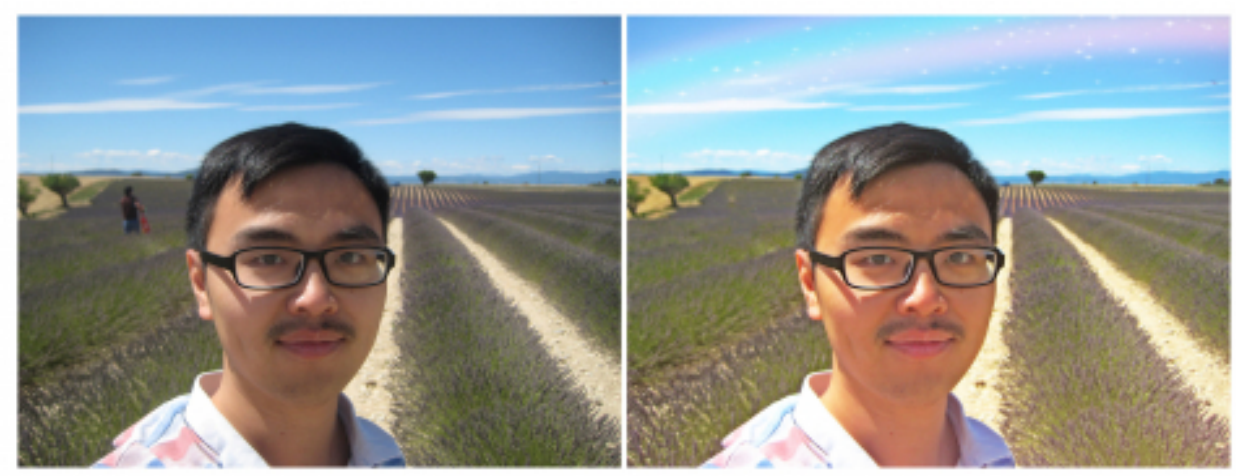

Source : Sina Microblog, 2016

Dans cette dernière phase, les échantillons montrent que ces touristes chinois - en particulier les femmes - personnalisent et " marquent » la destination grâce à l'infographie. Avec cette modification des photos de la destination, ils partagent leur expérience personnelle du voyage avec leurs réseaux sociaux en Chine.

Quand ces touristes asiatiques donnent un sens particulier à la destination «Provence ", ils le font en intégrant fortement les émotions positives de leur 
expérience, le contexte et les savoirs acquis avant le séjour et les références hors de la destination.

80 En bref, les touristes chinois pratiquent des activités touristiques assez uniformes et banales, mais leurs ressentis et attentes - perceptibles dans les commentaires - sont beaucoup plus complexes et peu adaptés à une offre touristique standardisée.

\section{Un « avant » et un « après »}

81 Les pratiques touristiques, voire d'appropriation, ne se limitent pas seulement à une action ou/et une activité pendant la durée du séjour touristique. Elles sont fortement influencées par les sensibilités des touristes d'avant-séjour » et impulsent des changements chez les touristes " après-séjour ", en particulier si nous considérons la sensibilité à travers la « pratique » comme une série d'activités en interaction. Les comparaisons permanentes avec le pays confirment également cette caractéristique de la pratique touristique. De ce point de vue, le tourisme est bien un processus interculturel en constante évolution.

Dans un cadre interculturel, les touristes s'approprient la destination en termes de nidification, d'exploration et de marquage. Ils consomment l'offre touristique de destination tout en continuant à partager et à développer une manière sensible de vivre ensemble en tant que Chinois. Ils adaptent et s'adaptent pour « survivre » dans un contexte particulier (voir tableau 3) selon divers degrés d'ouverture. L'exploitation des commentaires sur le réseau social met en évidence quelques caractéristiques des expériences touristiques. Les touristes mélangent et hybrident des pratiques et des comportements sociaux importés avec un cadre nouveau, une routine sécurisante et des habitudes - jeux, cuisine -, avec un inconnu plus perturbant, un collectif touristique chinois temporaire - le groupe -, avec des contacts et des échanges locaux.

\section{Tableau 3 : Attractivités sur la base des considérations des touristes}

\begin{tabular}{|l|l|l|}
\hline $\begin{array}{l}\text { Dans la destination, } \\
\text { quand les touristes... }\end{array}$ & Aiment le contexte & N'aiment pas le contexte \\
\hline $\begin{array}{l}\text { Aiment le lifestyle (mode } \\
\text { de vie) }\end{array}$ & $\begin{array}{l}\text { Ils restent et cherchent à vivre dans la } \\
\text { destination, comme les locaux }\end{array}$ & $\begin{array}{l}\text { Ils pratiquent le lifestyle de la } \\
\text { destination quand ils rentrent chez eux }\end{array}$ \\
\hline N'aiment pas le lifestyle & $\begin{array}{l}\text { Ils retoument fréquemment à la } \\
\text { destination pour visiter, mais conservent } \\
\text { leurs habitudes de vie quotidienne }\end{array}$ & $\begin{array}{l}\text { Ils peuvent simplement vivre une } \\
\text { expérience mémorable }\end{array}$ \\
\hline
\end{tabular}

Réalisation : les auteurs

Les habitants (en tant que culture locale) et les touristes (en tant que culture étrangère) sont tous des pratiquants (qui cherchent à vivre dans le contexte géographique) du site selon leur sensibilité et leurs modes de vie quotidienne. Il y a une différence de taille toutefois : les habitants sont les premiers arrivés et les touristes demeurent les éternels seconds.

\section{Des limites}

Notre démarche exploratoire comporte naturellement des limites. En termes de lourdeur de la procédure d'identification des commentaires d'abord, puisque le travail a été fait manuellement lors d'un suivi quotidien qui a permis d'identifier les commentaires de visiteurs chinois liés à l'expérience touristique. Nos conclusions ne peuvent donc pas être généralisées à l'ensemble des touristes chinois. Notre étude est par essence limitée aux membres et utilisateurs du réseau social Sina Microblog (Weibo). Une autre limite concerne la précision géographique, puisque la localisation des touristes est limitée à un site et ne permet pas toujours d'identifier un monument, une rue, un haut lieu. La composition de l'équipe de recherche, deux Chinois et un natif français, n'est sans doute pas encore suffisamment ouverte pour permettre une « compréhension culturelle » satisfaisante d'un phénomène aussi complexe. La présence d'un psychologue dans la suite des travaux constituera un avantage. 


\section{Conclusion. Vers une nouvelle intelligence touristique}

Cette première approche des pratiques et des ressentis in vivo des touristes chinois à partir de l'exploitation de leurs commentaires sur le réseau social Sina Microblog (Weibo) nous a permis de dresser une première typologie de comportements. L'approche interdisciplinaire par laquelle nous avons croisé des dimensions issues de la géographie (localisation...) et des sciences de gestion (nidification, exploration, marquage...) autour d'un objet commun (tourisme) devra être poursuivie et améliorée, avant de pouvoir passer à une modélisation, une automatisation des procédures sur des échantillons plus larges. Elle pourra être complétée par d'autres travaux sur des lieux différents, des moments variés et avec d'autres touristes dans d'autres contextes interculturels. Même en l'état actuel, cette exploration ouvre déjà de nombreuses perspectives dans une logique "d'intelligence touristique » où l'analyse de données (offre, demande, ressenti...) en temps réel est croisée avec l'animation permanente de " clusters touristiques », de plateformes de dialogue et d'innovation « visiteurs-acteurshabitants » vivantes privilégiant les rencontres et les échanges en face à face comme éléments de l'expérience touristique et garantie d'une meilleure cohabitation.

Devant les caricatures sur « l'idiot du voyage » (Urbain, 1991), les « manifestes » qui condamnent l'activité (Christin, 2017) et ses effets, les récentes manifestations « tourismophobes » dans de nombreuses métropoles (Venise, Amsterdam, Barcelone...) confrontées au " tourisme excessif » et l'explosion prévisible de cette activité à l'échelle mondiale, la connaissance des pratiques et des ressentis des touristes est une nécessité pour la cohabitation harmonieuse entre populations permanente et temporaire. Taleb Rifai, secrétaire général de l'Organisation mondiale du tourisme (OMT), remarquait, à l'occasion du Sommet interministériel de l'OMT en novembre 2017 : « Ce n'est pas la croissance qui est l'ennemie, ni l'affluence. Ce qui est essentiel, c'est la gestion durable, responsable et intelligente de la croissance. »

Cette nouvelle « intelligence touristique » partagée peut s'appuyer en partie sur l'exploitation de données issues de réseaux sociaux numériques comme Sina Microblog (Weibo). En ce sens, cette première approche du tourisme comme " expérience sensible » a permis de l'appréhender comme un «processus » complexe de relations avec l'environnement, une forme " d'être au monde » (Heidegger, 1986), plutôt que comme un simple déplacement, une « hybridation » plutôt qu'un exotisme.

Ces éléments pourraient être pris en compte dans l'exploitation et la création de " contenus » touristiques qui dépassent le simple passage de la vie quotidienne à l'offre touristique, et vice versa. Par rapport à une approche classique d'un phénomène normalisé de masse et à une analyse parfois caricaturale des comportement grégaires du touriste chinois, notre recherche met en évidence l'importance de la sensibilité humaine et des caractéristiques individuelles dans la construction des rapports aux espaces, aux temps et aux personnes qui caractérisent l'expérience touristique et sans doute plus largement nos nouveaux modes d'habiter polytopiques (Stock, 2006) et multi-scalaires.

Cette première approche sensible des situations touristiques redonne un sens à des mots aussi importants que " présence ", qui « n'est celle d'un soi que par l'ouverture à l'événement » (Maldiney, 1991), ou « exister », c'est-à-dire « avoir sa tenue hors de soi » dans l'ouverture.

Enfin, elle permet d'aborder et de concevoir le tourisme comme une situation " expérientielle » et " existentielle » (Schmitt, 1999), où le corps et l'affect sont mobilisés, et d'imaginer d'autres manières de cohabiter les espaces et les temps. En ce sens, réfléchir aux manières « d'habiter »- comme " mode de connaissance du monde » et « type de relations affectives loin d'une approche abstraite ou technocratique de l'espace » (Dardel, 1952) - en touriste ouvre des perspectives qui dépassent le seul domaine du tourisme pour embrasser l'ensemble de nos modes de vie contemporains.

91 Agapito, Dora, Júlio Mendes et Patrícia Valle, 2013, « Exploring the Conceptualization of the Sensory Dimension of Tourist Experiences ", Journal of Destination Marketing \& Management, vol. 2, no 2, p. 62 - 73. 
Arnould, Éric et Linda L. Price, 1993, « River Magic: Extraordinary Experience and the Extended Service Encounter », Journal of Consumer Research, vol. 20, no 1, p. $24-45$.

Atout France (Agence de développement touristique de la France), 2016, Les touristes chinois : comment bien les accueillir ? Paris, ODIT (Observation, développement et ingénierie touristiques) France.

Aubert-Gamet, Véronique, 1996, Le design d'environnement commercial dans les services : appropriation et détournement par le client, thèse de doctorat en sciences économiques, Université Aix-Marseille 3, Marseille.

Augoyard, Jean-François, 2010, Faire une ambiance, Bernin, La Croisée.

Banque mondiale, n.d., <http://donnees.banquemondiale.org/indicator/ ST.INT.ARVL?end=2016\&start=1995\&view=chart $>$, consulté le 21 mars 2017.

Batat, Wided et Isabelle Frochot, 2013, Marketing expérientiel, Paris, Dunod.

Bonnin, Philippe, 2002, " Manières d'habiter : l'étendue, l'espace, la ville », Communications, no 73, p. 5 - 9.

Bourdeau, Philippe et Liliane Bensahel, 2013, Fin (?) et confins du tourisme : interroger le statut et les pratiques de la récréation contemporain, Paris, L'Harmattan.

Carù, Antonella et Bernard Cova, 2003, "Approche empirique de l'immersion dans l'expérience de consommation : les opérations d'appropriation », Recherche et applications en marketing, vol. 18, no 2, p. 47 - 65.

101 Carù, Antonella et Bernard Cova, 2006, « Expériences de consommation et marketing expérientiel », Revue française de gestion, vol. 32, no 162, p. 99 - 114.

102 Christin, Rodolphe, 2017, Manuel de l'antitourisme, Montréal, Ecosociété.

103 Dardel, Éric, 1952, L’Homme et la Terre : nature de la réalité géographique, Paris, Éditions du CTHS (Comité des travaux historiques et scientifiques).

De Certeau, Michel, 1980, L’invention du quotidien, Paris, Gallimard.

105 De Souza Bispo, Marcelo, 2016, " Tourism as Practice », Annals of Tourism Research, vol. 61, p. 170 - 179.

106 Edensor, Tim, 2007, « Mundane Mobilities, Performances and Spaces of Tourism », Social \& Cultural Geography, vol. 8, no 2, p. 199 - 215.

107 Firat, A. Fuat, 2001, « The Meanings and Messages of Las Vegas: The Present of Our Future »,M@n@gement, vol.4, no 3, p. 101 - 120.

108 Fischer, Gustave Nicolas, 1981, La psychosociologie de l'espace, Paris, Presses universitaires de France.

109 Fischer, Gustave Nicolas, 1983, Le travail et son espace : de l'appropriation à l'aménagement, Paris, Dunod.

110 Fischer, Gustave Nicolas, 1992, Psychologie sociale de l'environnement, Toulouse, Privat.

111 Fornerino, Marianela et Agnès Helme-Guizon, 2006, " L’immersion dans une expérience de consommation vers une échelle de mesure », Cahiers de recherche du CERAG (Centre d'études et de recherches appliquées à la gestion), vol. 16, no 20, p. $42-57$.

112 Frochot, Isabelle et Dominique Kreziak, 2009, Étude de l'expérience touristique en stations de montagne, Chambéry, Savoie Mont Blanc Tourisme.

113 Gherardi, Silvia, 2009, « Practice? It's a Matter of Taste! », Management Learning, vol. 40, no 5, p. 535 - 55 o.

114 Gnoth, Jürgen et Xavier Matteucci, 2014, " A Phenomenological View of the Behavioural Tourism Research Literature », International Journal of Culture, Tourism and Hospitality Research, vol. 8, no 1, p. 3 - 21.

115 Gravari-Barbas, Maria et Marie Delaplace, 2015, " Le tourisme urbain 'hors des sentiers battus'», Téoros, vol. 34, nos 1 - 2. DOI:10.7202/1038815ar.

116 Guignon, B. Charles, 1984, "Heidegger's 'Authenticity' Revisited », The Review of Metaphysics, vol. 38, no 2, p. 321 - 339.

117 Gwiazdzinski, Luc, 2016, L'hybridation des mondes, Les territoires et les organisations à l'épreuve de l'hybridation, Grenoble, Elya.

118 Gwiazdzinski, Luc et Guillaume Drevon, 2014, « Des méthodes et des outils au service d'une nouvelle intelligence des mobilités », Netcom, Netcom and Communication Studies, vol. 28, nos 1 - 2, p. 5 - 12. 
Gwiazdzinski, Luc et Olivier Klein, 2014, « Du suivi GPS des individus à une approche chronotopique. Premières apports d'expérimentations et de recherches territorialisées », Netcom, vol. 28, nos 1 - 2, p. 77 - 106.

Heidegger, Martin, 1986, Etre et temps, Paris, Gallimard.

$\mathrm{Hu}$, Wenbo, Luc Gwiazdzinski et Wanggen Wan, 2016, " Les nuits de Shanghaï. Première approche spatio-temporelle à partir des réseaux numériques sociaux ", Netcom, vol. 30, nos 3 - 4, p. 181 - 206.

Ladwein, Richard, 2002, "Voyage à Tikidad : de l'accès à l'expérience de consommation », Décisions marketing, no 28, octobre-décembre, p. 53-63.

Ladwein, Richard, 2003 [2e éd.], Le comportement du consommateur et de l'acheteur, Paris, Economica.

Larsen, Jonas, 2008, « De-exoticizing Tourist Travel: Everyday Life and Sociality on the Move », Leisure Studies, vol. 27, no 1, p. 21 - 34.

Lucchini, Françoise, Bernard Élissalde et Sylviano Freire-Diaz, 2013, " Caractériser l'attractivité des quartiers urbains par les données de téléphonie mobile ", L’information géographique, vol. 77, no 1, p. 44 - 62.

Mackenzie, S. Houge et John H. Kerr 2013, « Can’t We All Just Get Along? Emotions and the Team Guiding Experience in Adventure Tourism », Journal of Destination Marketing \& Management, vol. 2, p. 85 - 93.

Maldiney, Henri, 1991, Penser l'homme et la folie, Grenoble, Jérôme Millon.

Obrador, Pau, 2003, "Being-on-holiday: Tourist Dwelling, Bodies and Place », Tourist Studies, vol. 3, no 1, p. 47 - 66.

130 Prohansky, Harold M., William H. Ittelson et Leanne G. Rivlin, 1970, Environmental Psychology - Man and His Physical Setting, New York, Holt, Rinehart and Winston.

131 Revue Espace, 2014, "Qu'est-ce qu'une 'expérience' ? », Revue Espace (Éditions touristiques européennes), vol. 320, p. 56 - 57.

Sans auteur (2016), « Festival d'Avignon: fréquentation en hausse malgré l'attentat de Nice », Ladepeche.fr, https://www.ladepeche.fr/article/2016/07/24/2390285-festivalavignon-frequentation-hausse-malgre-attentat-nice.html

Sansot, Pierre, 1985, La France sensible, Paris, Payot.

134 Schmitt, H. Bernd, 1999, Experiential Marketing: How to Get Customers to Sense, Feel, Think, Act and Relate to Your Company and Brands, New York, The Free Press.

Stock, Mathis, 2006, "L'hypothèse de l'habiter poly-topique : pratiquer les lieux géographiques dans les sociétés à individus mobiles », EspacesTemps.net, « Travaux », n.p., < <https://www.espacestemps.net/articles/hypothese-habiter-polytopique/>, consulté le 7 décembre 2016.

Thompson, J. Craig, 1998, « Living the Texts of Everyday Life: A Hermeneutic Perspective on the Relationships Between Consumer Stories and Life-World Structures », dans Barbara Stern (dir.), Representing Consumers, Londres, Routledge, p. $127-155$.

137 Tourisme Montréal, 2014, Le marché touristique de la Chine continentale, <http://www.octgm.com/toolkit/fr/statistiques/marche-chine-2014-fr.pdf>, consulté le 4 février 2017.

138 Urbain, Jean-Didier, 1991, L'idiot du voyage. Histoires de touristes, Paris, Payot.

139 Urry, John, 1992, " The Tourist Gaze 'Revisited' », American Behavioral Scientist, vol. 36, no 2, p. 172 - 186.

\section{Notes}

$1 \quad<$ http://www.marketing-chine.com/analyse-marketing/le-reve-des-touristes-chinois-laprovence>, consulté le 4 mai 2018.

2 Voir : <https://www.mediapart.fr/studio/documentaires/culture-idees/voyage-en-occidentavec-des-chinois-en-vacances> ; <http://www.dailymail.co.uk/news/article-4629748/Chinesetourists-spotted-cooking-meal-airport.html $>$

<http://www.rachellaudan.com/2011/01/chinese-tourists-dont-eat-european-food.html>, consultés respectivement les 18 avril 2017, 21 avril 2017 et 2 juillet 2017.

$3<$ https://www.economist.com/node/17722582>, consulté le 23 avril 2017. 
4 Centre national des ressources textuelle et lexicales, 2012, <http://www.cnrtl.fr/definition/adaptation>, consulté le 15 juillet 2018.

5 Voir <https://www.parisinfo.com/decouvrir-paris/guides-thematiques/organiser-un-weekend-a-paris/un-week-end-pour-decouvrir-paris-insolite/Des-suggestions-de-visites-insolites\%C3\%Ao-Paris>, consulté le 15 juillet 2017.

6 Association Marseille autrement, <https://www.20minutes.fr/marseille/924521-20120427autre-regard-ville>, consulté le 15 juillet 2017.

7 Toutes les citations tirées de publications anglaises sont nos traductions.

8 À ce sujet voir aussi Pons (2003), et Heidegger (1986).

\section{Table des illustrations}

Titre Tableau 1 : Quatre types de pratiques du tourisme

Crédits Réalisation : les auteurs

URI http://journals.openedition.org/teoros/docannexe/image/3542/img-1.jpg

Fichier image/jpeg, 212k

Titre Tableau 2 : Typologie expérientielle de pratiques touristiques

Crédits Réalisation : les auteurs

URI http://journals.openedition.org/teoros/docannexe/image/3542/img-2.jpg

Fichier image/jpeg, 140k

Titre Illustration $1:$ Interaction des touristes et des objets touristiques

Crédits Réalisation : les auteurs

URI http://journals.openedition.org/teoros/docannexe/image/3542/img-3.jpg

Fichier image/jpeg, 180k

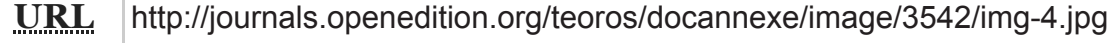

Fichier image/jpeg, 440k

Titre Tableau 3 : Attractivités sur la base des considérations des touristes

Crédits Réalisation : les auteurs

UيL http://journals.openedition.org/teoros/docannexe/image/3542/img-5.jpg

Fichier image/jpeg, 181k

\section{Pour citer cet article}

Référence électronique

Bule Liu, Luc Gwiazdzinski et Wenbo Hu, « Le tourisme comme expérience sensible », Téoros [En ligne], 38, 1 | 2019, mis en ligne le , consulté le 14 mai 2019. URL : http://journals.openedition.org/teoros/3542

\section{Auteurs}

Bule Liu

Géographe, Master 2 en tourisme, Institut de géographie alpine, Université Grenoble Alpes,

France : virgillbl@hotmail.com

\section{Luc Gwiazdzinski}

Géographe, enseignant en aménagement et urbanisme, Université Grenoble Alpes ; Professeur associé à Shanghaï University ; Chercheur UMR PACTE 5194 CNRS, Associé à l'EIREST (Paris 1, Panthéon Sorbonne) et au MOTU (Milan) ; luc.gwiazdzinski@univ-grenoble-alpes.fr

Wenbo Hu

Géographe, doctorant en aménagement et urbanisme, Université Grenoble Alpes, Laboratoire Pacte (UMR 5194 CNRS) ; wb.hoo@hotmail.com

\section{Droits d'auteur}




\section{(c)}

La revue Téoros est mise à disposition selon les termes de la Licence Creative Commons Attribution - Pas d'Utilisation Commerciale - Pas de Modification 4.0 International. 